Fecha de recepción: mayo 2021 Fecha de aceptación: junio 2021 Versión final: julio 2021

\section{Entre lo dicho y lo escrito: diálogo sobre diseño e innovación social desde una perspectiva sociosemiótica}

\author{
Dora Ivonne Alvarez Tamayo ${ }^{(1)}$
}

\begin{abstract}
Resumen: El diseño como proceso, producto, y acción, cuenta con un potencial transformador en las sociedades contemporáneas. En los últimos tiempos, las sociedades han enfrentado retos que convocan al despliegue de la capacidad intelectual -lógica y creativa-, al desarrollo tecnológico, a los procesos de reinterpretación y a nuevos acercamientos, que mejoren la calidad de vida de las personas y favorezcan sus interacciones en contextos y circunstancias específicas. El gremio del diseño se encuentra llamado a participar en procesos de innovación para reducir la distancia entre la generación de ideas y la concreción de las mismas, poniendo de manifiesto la necesidad de instaurar una cultura de la innovación social que constituya un marco de referencia para la formación profesional. En esta investigación se plantea un diálogo entre lo escrito y lo dicho respecto al diseño y la innovación social, considerando los principios del discurso social propuestos por Angenot. En primera instancia, se desarrolla un análisis sociosemiótico del concepto, a través de la revisión de literatura -lo escrito- para posteriormente comparar los discursos emitidos por una comunidad académica universitaria al respecto -lo dicho-, teniendo como base un estudio exploratorio cuantitativo. Resultado de este diálogo, se ofrece una definición de innovación social y se destacan los valores semánticos capaces de constituir una base de codificación para la instauración de una cultura de la innovación social y de su praxis. El análisis se desarrolla en una universidad privada de la ciudad de Puebla, México, mediante un enfoque socio-semiótico con base en los fundamentos relativos al discurso social de Marc Angenot, y la teoría del código de Umberto Eco.
\end{abstract}

Palabras clave: diseño - innovación social - instauración cultural - significación - discurso social - diálogo.

[Resúmenes en inglés y portugués en la página 68 ]

(1) Catedrática investigadora de la Universidad Popular Autónoma del Estado de Puebla desde 1999. Licenciada en Diseño Gráfico, Maestra en Procesos de Diseño y Doctora en Dirección y Mercadotecnia (UPAEP), mención honorífica por investigación. Posdoctorado en Ciencias Sociales en la Universidad Nacional de Córdoba, Argentina y una estancia internacional en Oklahoma State University. Pertenece al Sistema Nacional de Investigadores (SNI-CONACYT). Miembro de la Semiotic Society of America. 


\section{Introducción}

El discurso social es considerado evidencia de las creencias que justifica las acciones de los individuos. Extrapolándolo a la dimensión social, Angenot (2010, p. 82), explica que "no existe práctica que no se instituya sin el acompañamiento de un discurso que hable de ella legitimándola". Las manifestaciones discursivas que develan la cultura de una sociedad, se acompañan de convenciones validadas por el grupo social en cuestión. Éstas constituyen, además, la base del aprendizaje de códigos de diversa índole, que regulan las interacciones pero que, a su vez, predisponen el pensamiento de los sujetos ya sea para seguir las reglas o para romperlas.

Dado que la innovación simboliza la esperanza de bienestar social ante las circunstancias que la humanidad enfrenta en el siglo XXI (Brunet, 2013), se ha puesto en evidencia la necesidad de fomentar en los individuos y en las sociedades el potencial creativo y la mentalidad innovadora. El diseño, dada su orientación a la solución de problemas y a la generación de satisfactores que faciliten las interacciones del ser humano en múltiples contextos, se suma a la búsqueda de mejorar las condiciones y la calidad de vida, ofreciendo respuestas diferentes y viables ante los problemas contemporáneos. En su ejercicio, es posible percibir una orientación quasi-natural a la innovación social. Sin embargo, en los procesos formativos se requiere generar las condiciones y ambientes que favorezca el desarrollo de una mirada consiente y deliberada hacia ella, reconociendo la importancia de la instauración de una cultura que permita "respirar" innovación social. Este marco de referencia permitiría permear en el perfil de egreso a un diseñador-innovador, capaz de manifestarse como agente de cambio. Sin embargo, en el diálogo cotidiano, las expresiones asociadas a los temas de innovación, emprendimiento e innovación social, presentan diferentes acepciones, sentidos y matices, poniendo de manifiesto el carácter polisémico del término.

El discurso social es lo que se dice, se escribe y se produce públicamente; se trata de sistemas genéricos, repertorios y reglas que en una sociedad dada organizan lo decible (Angenot, 2010), develando la forma en que la colectividad expresa su conocimiento a través del lenguaje. Los discursos se producen bajo la influencia de ideas generales, apreciaciones, perspectivas, y definiciones de otros (Bajtín, 1978, p. 100 citado en Angenot, 2010). Lo que se dice está inscrito en la esfera de influencia de una doxa, es decir, de la opinión generada a partir de la experiencia, maleable, e impersonal y a su vez necesaria, que no ofrece certeza, pero que se manifiesta como una creencia razonable. En lo decible, se encuentran los lugares comunes y los factores que provocan la aceptabilidad del discurso -o hecho social-. La enunciación de un discurso no es aleatoria ni inconsciente; como hechos históricos, nacen, se alteran y descomponen en el tiempo. La identificación, descripción y explicación de las regularidades en un tiempo determinado son materia de análisis del discurso. Las regularidades permiten identificar los hechos sociales que están dotados de poder social, para imponerse ante una colectividad e interiorizarse en las conciencias de los individuos que la conforman (Angenot, 2010).

Esta investigación se organiza en tres partes: a) la revisión de la literatura para identificar la forma en que el concepto de innovación social ha sido documentado, b) la identificación de los cuadros de significación de los miembros de una comunidad académica 
mediante el análisis componencial y discursivo, y c) la comparación de los hallazgos para identificar unidades culturales que develen los cuadros de significación como base para la instauración de una cultura de la innovación social.

\section{Contexto}

El siglo XX, mostró un despliegue de desarrollo tecnológico, aunado a un crecimiento demográfico que requirió desarrollar nuevos esquemas de organización laboral y de configuración social que respondían a corrientes ideológicas polarizadas en los bloques capitalista y socialista. En el siglo XXI, la tecnología informática y de comunicaciones producen cambios vertiginosos en el funcionamiento de las organizaciones y en las dinámicas del individuo en comunidad. La economía es impulsada por la innovación y el conocimiento en esquemas competitivos de renovación constante; el campo laboral demanda profesionales con capacidades técnicas, formación práctica, habilidades directivas y espíritu emprendedor (Observatorio Laboral, 2012).

Diversos organismos se han enfocado en promover la innovación -en un sentido amplioy la innovación social de manera específica. Tal es el caso de ASHOKA (2017), la red más grande de emprendedores sociales a nivel mundial; la Comisión Económica de América Latina y el Caribe -CEPAL- (Rodríguez y Alvarado, 2008), Endeavor (2017), organismo que lidera el movimiento de emprendimiento de alto impacto y Enactus (2017), comunidad que fomenta el progreso de las comunidades mediante la acción positiva de los negocios. La ciudad de Puebla ha sido reconocida por la Organización de las Naciones Unidas para la Educación la Ciencia y la Cultura, UNESCO (2016) como Ciudad Creativa en la categoría de Diseño.

“Todo debate en un sector determinado, por más ásperos que sean los desacuerdos, suponen un acuerdo anterior sobre el hecho de que el tema que se trata existe" (Angenot, 2010, p.43). Ante las circunstancias actuales, los debates en torno a la innovación social han cobrado relevancia en tanto que existe como fenómeno social que se encarna en la agenda pública mundial. La comprensión del tema no se ha agotado, probablemente por las condiciones cambiantes que la propia cultura experimenta.

Esta investigación se situa en una universidad privada con sentido humanista, que promueve la formación de profesionales y la incidencia de su comunidad académica en la sociedad inmediata mediante sistemas de auténtica pertinencia social. El objetivo es contribuir a la reordenación del mundo de lo social, de lo económico y de lo político, promoviendo y generando valor al servicio del hombre (UPAEP, 2012), y asumiendo el compromiso de formación para un liderazgo transformador. 


\section{Lo escrito}

La innovación social se presenta como una temática cuyo conocimiento parcial no parece ofrecer dudas dado que el mundo habla de ellos, pero en el día a día, el uso del término presenta lagunas, desacuerdos y en muchas ocasiones falta de claridad. En términos de Angenot (2010) los discursos en cuestión producen un rumor social. Para el abordaje del concepto de innovación social, se partirá de la noción de innovación, la categoría superior en la que se inscribe, y con la cual — evidentemente-comparte rasgos generales. Ambos términos pertenecen a la tópica y funcionan como presupuestos colectivos para fundar los discursos en torno a ellas.

El estudio de la innovación ha despertado el interés de diversas disciplinas del conocimiento. La ciencia económica ha liderado la difusión de los llamados "estudios de innovación" los cuales requieren del carácter, al menos interdisciplinar, para comprender su dimensión social e impacto (Brunet y Pizzi, 2012). Así pues, la sociología se incorpora al estudio de la innovación en carácter de necesario. El concepto de innovación ha sido abordado desde diferentes perspectivas destacando, desde la visión económica los aportes de Schumpeter y su teoría de la destrucción creativa, el enfoque de sistemas y la construcción social de la innovación.

Schumpeter (2003), por ejemplo, sostenía que la innovación tenía que ver con la capacidad de hacer cosas fuera de la rutina, pero que invariablemente enfrentarían resistencia al cambio, puesto que un producto de la innovación podría introducir rupturas a las formas rutinarias de proceder. La innovación, paradójicamente, se convertiría en rutinaria con el tiempo, esbozando el ciclo interminable de la innovación. Por su parte, Baumol (2002) la refiere como un proceso permanente, recurrente y continuo, vinculado a los entornos competitivos de la economía globalizada en búsqueda de nuevas formas de organización, que impacten en la eficiencia de las empresas.

La innovación es definida, desde la perspectiva sistémica, como producto de un sistema de relaciones y procesos orientados a la cooperación y a la competencia. El sistema se compone de agentes, roles y reglas que afectan los insumos, las actividades, los mecanismos y los resultados (Edquist y McKelvey, 1997).

Desde una óptica constructivista-sociológica, se define como un proceso no lineal de producción de nuevas respuestas a necesidades, cuya importancia recae en la interacción de múltiples agentes — no sólo tecnológicos- y a la doble implicación entre investigación y aplicación. Esta perspectiva surge en controversia con modelos clásicos de innovación de tradición tecnológica lineal (Brunet y Pizzi, 2012).

$\mathrm{Al}$ analizar el contexto de la educación para la innovación en México, Martínez (2017) presenta algunas competencias para la innovación en relación a formas de hacer las cosas de manera diferente: la introducción de un nuevo bien o de una nueva calidad, un nuevo método de producción o nueva forma de manejar comercialmente la mercancía, la creación de una nueva organización y la conquista de una nueva fuente de aprovisionamiento. En este orden de ideas, la creatividad es parte del proceso fundamental para el desarrollo de la innovación. En cada una de las expresiones de la innovación propuestas, el común denominador es la novedad. 
Se consideran agentes de la innovación a organizaciones innovadoras, al empresario innovador y el emprendedor, cuya conducta tiene que ver con la creación de condiciones institucionales y de la formación y mentalidad emprendedora de sus colaboradores para promover la innovación (Brunet, 2013).

Aunque, de acuerdo con Cisternas y Kast (2014), en tanto que la innovación involucra necesariamente personas y organizaciones, toda la innovación es social, existen subcategorías y una de ellas es la innovación social, que comparte el espectro tipológico con la innovación tecnológica, de negocios, entre otras. La ecuación I+S tiene como meta alcanzar objetivos sociales, y su foco en mejorar las condiciones de vida de las personas generando valor.

La innovación social es un concepto ligado al poder de las sociedades, las cuales enfrentan retos que cuestionan las estructuras sociales tradicionales y las formas de operarlas, para encarar los retos contemporáneos. La innovación social es un tipo de innovación que surge "como la gran solución a los problemas sociales, económicos y medioambientales" (Alonso-Martínez, González y Nieto, 2016, p.121). El concepto está intrínsecamente relacionado con el bienestar social ante el deterioro del estado de bienestar del que es objeto un alto porcentaje de la población mundial.

La Comisión Europea (Hahn y Andor, 2013) la define que el desarrollo e implementación de nuevas ideas - productos, servicios y modelos- son aquellas orientadas a satisfacer las demandas y necesidades sociales, creando relaciones y colaboraciones con la sociedad.

La innovación social también tiene la acepción de estrategia de cambio, transformación y emprendimiento. En Europa, por ejemplo, la estrategia H2020 considera a la innovación social uno de sus pilares fundamentales, y la define como proceso y producto que tiene como objetivo final el logro de un desarrollo sostenible, eficiente, justo y ecológico para satisfacer las necesidades sociales y generar valor social (Alonso-Martínez, et al., 2016, p.122).

Se la considera también como el fundamento de un nuevo modelo de negocio que combina la rentabilidad con la responsabilidad social, orientado a la creación de sociedades híbridas que combinen objetivos sociales y económicos (Alonso-Martínez, et al., 2016). Este concepto no debe confundirse con negocios sociales, mercadotecnia social o responsabilidad social empresarial. Tampoco debe verse desde una perspectiva altruista o subsidiaria, puesto que este tipo de innovaciones requieren de ser sostenibles. La principal diferencia radica en su fin primario; las innovaciones están orientadas al bienestar social en sus fines y en sus medios (Hahn y Andor, 2013). El concepto de emprendimiento social suele aparecer cercano en el mapa semántico de la innovación social, con la salvedad de que las innovaciones sociales pueden generarse por empresas independientemente del objetivo de la misma (Alonso-Martínez, et al., 2016).

La Comisión Económica para América Latina y el Caribe (Rodríguez y Alvarado, 2008) señala que las innovaciones en el campo social a menudo surgen en condiciones adversas en los entornos donde ni el sector público ni el mercado han logrado responder a las demandas de la población. En este contexto, la innovación social es definida como proceso social que parte de la invención o la creatividad, y que mediante la aplicación localizada se convierte en innovación, cambiando el rumbo de tendencias, productos, procesos y técnicas con fundamento e impacto en la sociedad de la que surge y a la que sirve. 
De acuerdo con Córdoba-Cely, Villamarín y Bonilla (2014), las diferentes acepciones de innovación social muestran que se trata de un término polisémico, en pleno proceso de construcción. Su sentido depende del enfoque disciplinar y de la particularidad de la realidad que se busca transformar. Conceptos como la creatividad, la responsabilidad y la participación social destacan entre sus premisas base.

Teniendo en cuenta una acepción amplia del diseño, de acuerdo con Ortiz (2016), el alcance de las disciplinas orientadas al diseño se ha diversificado, evolucionando de la concepción de su ejercicio en aspectos formales, materiales y tecnológico-productivos a la búsqueda de impactar en los comportamientos que promueven sostenibilidad, emociones y experiencias en respuesta a los desafíos sociales contemporáneos.

Las áreas profesionales orientadas al diseño son consideradas como áreas creativas, no sólo coloquialmente, sino en su clasificación de competencia económica. La UNESCO (s/f) desde su vocación educativa, declara que forman parte de la industria creativa y cultural "aquellos sectores de actividad organizada que tienen como objeto principal la producción o la reproducción, la promoción, la difusión y/o la comercialización de bienes, servicios y actividades de contenido cultural, artístico o patrimonial". En esta concepción, la UNESCO enfatiza no sólo el proceso creativo, sino su relevancia en la cadena productiva y de difusión, por lo que incluye al diseño gráfico y la publicidad como elementos decisivos de ella.

Sin embargo, creatividad e innovación son términos que, en el ámbito de los negocios, la educación y la cultura suelen definirse como sinónimos. Es necesario hacer una precisión fundamental si se pretende instaurar una cultura de innovación que responda a las necesidades de la persona y las sociedades. A saber: la creatividad es una capacidad humana, una competencia y un proceso mental de generación de nuevas ideas realizando conexiones que ponen en juego el intelecto y la emoción, pero que, para responder a los problemas emergentes en entornos cambiantes, necesita ser implementada. De allí que la innovación sea considerada la aplicación de la creatividad, agregando valor a las diferentes esferas de la vida humana (Alvarez, Croda, Paredes y Domínguez, 2017). Esta perspectiva, enfatiza la necesidad de desarrollar la capacidad innovadora y reducir la distancia entre la generación de la idea y la concreción de la misma. Particularmente en el ámbito del diseño, si se pretende formar profesionalmente a un diseñador-innovador que se constituya en agente de cambio, se requiere instaurar una cultura de la innovación social de manera estratégica, que sirva de marco de referencia para la comunidad académica.

De acuerdo con Duarte y Cortés (2020, p.711) "El diseño para la innovación social se enfoca en la creación e implementación de soluciones que fomentan mejores condiciones sociales, proyectadas en comunidades, ciudades o regiones a través de la innovación, la conciencia social y la equidad". El concepto de innovación social incide en un amplio espectro que incluye el de la creatividad social, creatividad entendida como un proceso de reducción de incertidumbre social. Asimismo, este concepto apela a la incorporación de las disciplinas que conforman la categoría de industrias creativas y culturales (ICC), y a la realización de proyectos de innovación, investigación y desarrollo contribuyendo a la productividad empresarial mediante productos y servicios de impacto social y cultural (Córdoba-Cely, Villamarín y Bonilla, 2014). 
Un enfoque del diseño para la innovación social, contempla la creación de soluciones a la luz de una lógica del crecimiento humano, en contraste con una lógica de mercado. La producción de ideas originales y el uso de herramientas prácticas para concretarlas, se llevan a cabo reconociendo el rol social del diseño y su capacidad propulsora de cambio ante escenarios complejos (Ortiz, 2016). Esta perspectiva implica, que en su orientación pragmática, la mirada del diseñador se dirije al bien común, sin perder de vista la sostenibilidad de sus respuestas ante los retos, necesidades y expectativas que emergen en los entornos sociales. La intuición del impacto social que las intervenciones de diseño pueden tener, ha estado latente a través de la historia del diseño. Sin embargo, es en la actualidad que se ha tomado mayor conciencia de su rol protagónico como agente de cambio e influencia. Desde esta perspectiva, de acuerdo con Manzini, (2015), el diseñador puede desempeñarse como un agente de cambio, un facilitador de la innovación social, un activista, un estratega, y un promotor cultural. Para ello, recurre a su concepción de los procesos de diseño centrados en la persona, el diseño de experiencias, el Design Thinking y el co-diseño entre otros.

La cultura, entendida como el conjunto de símbolos y objetos aprendidos, compartidos y transmitidos de una generación a otra por miembros de una sociedad, es una gran fábrica de experiencia, significación y acción, en la que los individuos interactúan dentro de las reglas de un marco de referencia en que se manifiestan prácticas, sentimientos, pensamientos, patrones de conducta e interpretaciones (Arnould y Thompson, 2005). El individuo es concebido como un agente interpretativo significador-creador. Para Eco (2005, p.51), la cultura no puede reducirse sólo a fenómenos de comunicación y significación, sin embargo, "la cultura en su conjunto puede comprenderse mejor, si se la aborda desde un punto de vista semiótico".

De acuerdo con San Martín (2015), la instauración de una cultura exige, ante todo, una instauración nueva de sentido. La cultura en tanto sistema de significados unificados y compartidos por los miembros de una sociedad, no puede reducirse sólo a fenómenos de comunicación y significación, pero puede comprenderse mejor si se le analiza desde una perspectiva semiótica (Eco, 2005). Siguiendo a Eco (2005) la semiótica estudia los procesos culturales como fenómenos de comunicación —-mediados por discursos-; los signos son una fuerza social además de reflejar las fuerzas sociales. Para analizar los cuadros de significación de la innovación social, se debe comprender que una expresión es capaz de transmitir contenidos distintos, promoviendo la comprensión del discurso en varios niveles (Eco, 2005). Para desambiguar el sentido, el contexto ofrece referencias y el código el soporte regulatorio del sistema convencional que la comunidad comparte para producir e interpretar discursos.

Los signos que configuran los discursos son unidades culturales organizadas en estructuras sintácticas, semánticas y pragmáticas como campos y ejes semánticos. Las denotaciones identifican la expresión en relación a un contenido de primer grado que sirve de base para connotaciones o significados sucesivos.

No basta con que el creador establezca el nuevo sentido; el sentido a introducir requiere de sedimentarse y debe ser asumido por los demás. Para que el sentido propuesto sea asumido por otros individuos en sociedad, debe cumplir con alguna de estas características: ser congruente, adecuado con la serie de remisiones en la que se integra, cumplir una función 
para la que no había otro sentido, o mostrar que es necesario; de lo contrario no se logrará el proceso de instauración cultural.

Para promover la instauración cultural de la innovación social, se requiere de un sistema regulador para establecer las bases de la producción de formas discursivas concretas y argumentos que se construyen con base en el sistema de creencias. "La hegemonía, es un conjunto de mecanismos unificadores y reguladores" (Angenot, 2010, p.31), que da cuenta de repertorios y reglas en relación a lugares comunes -topoi- desde los cuales se establecen posiciones de influencia; aunque opera contra lo aleatorio, lo centrífugo y lo marginal, constantemente está en vías de renovación y transformación mediante el refuerzo en comunidad.

\section{Lo dicho}

De acuerdo con Angenot (2010) en lo decible se encuentran los lugares comunes y los factores que provocan la aceptabilidad del discurso que el autor considera como hecho social. Desde esta perspectiva, reconocer los cuadros de significación presentes en los discursos de una comunidad académica permite recuperar los lugares comunes que favorecerían la instauración de una cultura de innovación y fungirían como base estratégica para la introducción de nuevos discursos que den forma y dirección a los esfuerzos de formación. Así, el tratamiento del discurso social que Angenot (2010) propone, ofrecerá la posibilidad de conocer la forma en que la comunidad objetiva su conocimiento mediante el lenguaje, que devela sistemas genéricos, repertorios y reglas de lo decible en sociedad. Dado que la emisión de discursos parte de influencias -ideas, aportes, apreciaciones, conceptos y perspectivas provenientes de otros-, la doxa u opinión generadas a partir de la experiencia constituirá la materia de análisis que, si bien no ofrece certezas, da cuenta de una creencia social compartida y plausible.

Angenot (2010) sugiere la identificación de dominancias interdiscursivas. La dominancia de ciertos hechos semióticos en forma y contenido se produce por la interacción de los discursos, los intereses que los sostienen y la necesidad de pensar colectivamente la novedad, tomando en cuenta las significaciones inmanentes a las diversas prácticas y a las creencias que las movilizan.

Se trata de un estudio exploratorio, no experimental, comparativo, teniendo como base para el análisis de datos la perspectiva semiótica sobre el discurso social de Marc Angenot y la Teoría del Código de Umberto Eco. Las concepciones sobre innovación social provenientes de la literatura fueron sometidas a análisis para reconocer el campo semántico. En un segundo momento, se realizó una encuesta trasversal a la comunidad estudiantil universitaria considerando la participación de estudiantes de diferentes programas académicos. Finalmente, este mismo instrumento se aplicó a estudiantes del programa de licenciatura en Diseño Gráfico y Digital. El contexto del estudio fue una institución de educación superior particular, ubicada en Puebla, México. Los instrumentos de recolección de datos fueron aplicados durante la realización de eventos cuya temática estaba relacionada con el Ecosistema de Emprendimiento e Innovación. El proceso de recopilación de datos 
se efectuó mediante un cuestionario auto-administrado. Los datos recolectados fueron analizados desde una perspectiva socio-semiótica, tomando en cuenta las siguientes categorías de análisis: regularidades, discrepancias, dominancias en el plano de la expresión y del contenido, facilitadores y bloqueadores para la instauración cultural. Lo dicho y lo escrito se sometió a comparación.

El análisis de las definiciones provenientes de la literatura especializada en torno al concepto de innovación social incluyó la revisión de 55 documentos analógicos y digitales. Para el estudio de campo, se realizó un muestreo por conveniencia en dos grupos de la población. a) En la aplicación de la encuesta a la comunidad académica conformada por diversos programas educativos se logró una muestra de 564 participantes. La muestra contó con la representación de 41 áreas disciplinares de formación profesional. b) La muestra de la encuesta aplicada a estudiantes del programa de Diseño Gráfico y Digital estuvo conformada por 76 participantes.

$\mathrm{Al}$ analizar los textos académicos que abordan el tema de la innovación social, se detectaron como regularidades que la innovación es interdisciplinar, se relaciona con la generación de algo nuevo en tanto productos y procesos, también con la introducción de mejoras y la aplicabilidad. La innovación aporta valor y se gesta mediante la creatividad aplicada, dando paso a los productos de la innovación. La innovación está conectada con los procesos productivos, económicos y de globalización. Respecto a la innovación social, la regularidad es el involucramiento de personas y organizaciones, se orienta a alcanzar objetivos sociales, y se enfoca a la mejora de la calidad de vida de las personas. Sus productos están orientados al bienestar social en sus fines y medios.

Las discrepancias principales respecto a la innovación radican en el enfoque desde el que se aborda y las diferencias de sus acepciones como proceso, producto y estrategia. También difieren sus métodos y objetivos, destacando una marcada diferencia entre la visión lineal y la sistémica. Sobre la innovación social, las discrepancias se centran en la perspectiva de los agentes. Su aplicabilidad puede variar en tanto impacto global o local. Las condiciones adversas del entorno pueden ser detonantes de la innovación social pero también pueden bloquearla.

Las dominancias son: la novedad, la relación creatividad-innovación, el innovador como agente de cambio y la orientación de la innovación social hacia el bienestar social. La palabra más utilizada en innovación es /nuevo/ y en innovación social /bienestar/, /necesidades/ y /social/. La denotación de innovación social es: proceso de generación de soluciones a necesidades sociales que promuevan bienestar y calidad de vida de las personas; y entre sus connotaciones aparecen los productos, las interacciones, el impacto social, el emprendimiento social, los negocios sociales y las innovaciones sostenibles. Tomando como base esta definición de innovación social, al revisar la literatura, también se estableció el campo semántico de la innovación social puesto en tinta y papel por especialistas en el tema. En él se identificó una mancuerna clave para la comprensión del fenómeno de la innovación social: la creatividad y la responsabilidad. Ambas encuentran aplicabilidad en contextos específicos, impulsadas por personas y organizaciones, ligadas al emprendimiento, y a la búsqueda de mejoras, modificaciones y alternativas en el terreno social y económico. Lo social es el corazón del término que, de manera transversal, enfatiza los significados de estructuras, bienestar, problemas y cambio. 
Los facilitadores de la innovación social son la participación de gobiernos, instituciones educativas, sociedad y empresa, la disposición favorable al cambio, la generación de políticas públicas, el conocimiento, la voluntad y las capacidades personales en atención a las necesidades sociales y del entorno. Los bloqueadores son las condiciones contextuales que producen inviabilidad, resistencia al cambio, desconocimiento, rumor y desacuerdo, limitantes de tipo económico, y falta de visión hacia la sostenibilidad.

El análisis componencial de las unidades culturales (plano de la expresión en relación con el plano del contenido a nivel denotativo y connotativo) en el campo semántico de la innovación social, permitió reconocer que los participantes han estado en contacto con el lenguaje formal promovido al interior de la comunidad académica, ya sea en clases o eventos relativos al tema. La comunidad muestra conocimiento al menos liminal.

Las regularidades identificadas son: la alusión a la persona como agente de cambio, la continua aparición de la tecnología ante la expresión /innovación/, la innovación social relativa a la mejora de la calidad de vida y el beneficio social. La denotación de innovación social es hacer algo nuevo para la gente. La palabra dominante es ayudar. Aunque creatividad e innovación no son sinónimos, existe un nexo causal evidente, al menos para la comunidad respondiente, que en ocasiones los lleva utilizar estos términos de manera indistinta. Más que discrepancias significativas, se reconocen énfasis o matices en torno a la aparición del concepto de bien común en el cuadro de significación de la innovación y el de emprendedor. Las dominancias son la innovación en relación con lo nuevo, la creatividad asociada a la innovación, el emprendedor como líder, la innovación social en relación a la sociedad, y la ayuda. La palabra más utilizada en la comunidad académica es /nuevo/ seguida de / creatividad/, /persona/, /cambio/ y /emprender/.

La denotación de innovación social es: la generación de ideas nuevas para la solución de problemas sociales que produzcan un cambio que impacte positivamente a la sociedad. Las connotaciones incluyen búsqueda del bien común, satisfactores sociales, ayuda, bien social y cambio.

Los participantes del estudio adscritos a Diseño Gráfico y Digital, en términos de regularidades, subrayan los conceptos de beneficios sociales y solución de problemas. Explícitamente se visualizan como agentes de cambio y, por tanto, solucionadores de problemas. Al analizar las respuestas, se observó que hay discrepancias respecto a la relación del concepto de innovación social con el componente empresarial, incluso llega a pasar desapercibido y poco se menciona el concepto de bienestar.

La muestra coincide en la mención reiterada del término creatividad, siendo éste el de mayor dominancia. Suele suceder que las palabras creatividad e innovación son utilizadas como sinónimos, o en su defecto, vinculadas como pares de una metonimia de causa por efecto. $\mathrm{Al}$ analizar las denotaciones ofrecidas por los estudiantes de Diseño Gráfico y Digital la definición integrada del concepto innovación social es: proceso de proponer soluciones creativas a problemas sociales. A nivel connotativo, se contemplan los conceptos de creatividad, diseño centrado en el usuario, satisfactores sociales y responsabilidad social. 


\section{Diálogo entre lo dicho y lo escrito}

Al comparar lo dicho por la comunidad académica diversificada y la comunidad de estudiantes de Diseño Gráfico y Digital, se identifican algunos elementos coincidentes, y otros que son particulares de la experiencia del usuario en su ámbito académico específico. Considerando la estructura de la función semiótica constituida por el plano de la expresión y el plano del contenido para realizar el análisis componencial, en el caso de la comunidad académica, la denotación, gira en torno a la solución de problemas, la generación de cambios y el impacto positivo. Las connotaciones o contenidos en segundo grado, incorporan los términos de búsqueda de satisfactores para el bien social, ayuda, bien común y cambio.

Por su parte, denotaciones ofrecidas por los estudiantes de Diseño Gráfico y Digital incluyen los términos de /proceso/, /soluciones creativas/ y /respuesta a problemas sociales/. A nivel connotativo, se contemplan los conceptos de creatividad, diseño centrado en el usuario, satisfactores sociales y responsabilidad social.

Para la instauración de una cultura de la innovación social en términos de regularidad tanto comunidad universitaria como estudiantes de Diseño Gráfico y Digital identifican los siguientes facilitadores: liderazgo, habilidad personal, trabajo en equipo, tecnología y visión empresarial. Respecto a los bloqueadores, son regulares las respuestas de resistencia al cambio y la percepción de que la innovación social requiere un alto nivel de esfuerzo. Los sujetos de ambas muestras enfatizaron, de manera recurrente, la vocación de la persona por ayudar a otros y mejorar la calidad de vida, expresaron preocupación por las personas, y el reconocimiento de su capacidad para promover cambios positivos en la sociedad, revelando en su lenguaje los constructos simbólicos que predisponen favorable o desfavorablemente al individuo hacia la instauración de una cultura de la innovación social.

Al comparar lo escrito y lo dicho, las regularidades recaen en la generación de algo nuevo, la introducción de mejoras, la creatividad, el involucramiento de las personas y el bienestar social. Discrepan en la construcción de los conceptos; lo escrito revela una reflexión profunda y fundamentada, aunque inacabada, a diferencia de la construcción empírica del código de los respondientes.

En lo escrito se ha manifestado con mayor precisión la influencia de los factores contextuales y circunstanciales, en lo dicho se expresa la intuición de la importancia del contexto. Sin embargo, el discurso de la comunidad ha hecho especial hincapié en la vocación de la persona por ayudar a otros, a mejorar su calidad de vida, y en sus respuestas manifiestan una preocupación por la persona y el reconocimiento de la capacidad que ésta tiene para promover un cambio positivo en la sociedad. Las dominancias convergen en torno a lo nuevo. En los discursos de la comunidad se reconocen como facilitadores de la innovación social el liderazgo, la capacidad personal, el trabajo en equipo, la tecnología y la visión del emprendedor. Los bloqueadores coinciden con la resistencia al cambio y la percepción de que la innovación social requiere un alto grado de esfuerzo. Cabe mencionar que los respondientes hacen uso recursivo de los términos conforme a los campos semánticos compartidos para explicar los términos. 


\section{Conclusión}

El diálogo entre lo dicho y lo escrito permitió integrar una definición de innovación social: la aplicación de la creatividad a situaciones específicas en contextos determinados, agregando valor en diferentes esferas de la vida humana y causando impacto positivo en la cultura, el individuo y las sociedades. Al comparar las estructuras semánticas de lo dicho y lo escrito se puede observar que, aunque los miembros de una comunidad no tengan dominio total de un concepto, en la medida que participan de las interacciones sociales aprehenden e incluyen el uso de las expresiones en sus conversaciones cotidianas de forma empírica o intuitiva. De manera que, un ambiente apropiado para la socialización de los términos ayuda a las personas a interiorizar el concepto asociado a la práctica.

Dado que el lenguaje devela las construcciones simbólicas que subyacen predisposiciones de los individuos, si se pretende impulsar una cultura de la innovación social, es necesario analizar el fenómeno de la construcción de los significados en torno a ella. Una vez traídos a conciencia los conceptos compartidos, se puede reforzar el sentido de comunidad.

En esta investigación se fluctuó entre lo escrito y lo dicho en búsqueda de un balance conceptual que permitiera incorporar los discursos formales sobre la innovación social al código de una comunidad académica para su instauración cultural. A nivel metodológico, se vinculó el análisis componencial del código y el análisis del discurso social para reconocer el imaginario colectivo. Se hizo patente que la innovación social implica una visión integral que permita que los hallazgos sean difundidos, las innovaciones implementadas y el conocimiento expresado en términos asequibles a la población, de manera que pueda incorporarse a los códigos operantes de la comunidad.

La base para la instauración de una cultura de la innovación es el conocimiento del sistema de creencias y los códigos base de una sociedad. La transformación integración y autorregulación del código como sistema, es necesaria para la instauración de dicha cultura, porque permite introducir elementos de adhesión y acuerdo pese a las distintas visiones del mundo. Este estudio abre la posibilidad de extender la línea de investigación con pares en Latinoamérica con la intención de identificar estadios simbólico-conceptuales y elementos contextuales compartidos.

Acerca del diseño, el concepto de innovación social pone en evidencia su injerencia en el desarrollo de innovaciones con responsabilidad social. Para el diseño, representa una base conceptual para instaurar una cultura en torno a ella, y a través del diseño es posible hacerla vida en forma sostenible, situada y pragmática, dando sentido al ejercicio profesional y extendiendo sus alcances como factor de influencia en las interacciones humanas.

\section{Agradecimientos}

Al programa de Fondo Concursable UPAEP, al Departamento de Artes y Humanidades y a la Facultad de Diseño de la UPAEP, a la Dra. María Teresa Dalmasso, al Dr. Marc Angenot, Al Ing. Rubén Gatica, a los pares investigadores y a los coordinadores del $13^{\circ} \mathrm{Ciclo}$ del 
posdoctorado CEA-UNC: Dr. Marcelo Casarín, Dra. Dora Barrancos, Dra. Pampa Arán y Dr. Renato Ortiz, al Grupo de Investigación Emprendimiento e Innovación Social: Dra. Gabriela Croda, Mtra. Olivia A. Paredes, y Mtra. Ana Laura Domínguez.

\section{Lista de referencias bibliográficas}

Alonso-Martínez D., González, N. y Nieto, M. (2016). La percepción de la innovación social en España”, En Aguado, J, García-Parada, G. Hernández, M. y Rodríguez, L. (Eds.). Global Entrepreneurship Monitor. Informe GEM España 2016. (pp.121-132). Santander: Editorial de la Universidad de Cantabria.

Alvarez, D., Croda, G., Paredes, O. y Domínguez, A. (2017). ¿Creativación e innovatividad? Un estudio exploratorio. En Murillo, L. y Ceja, S. (Ed.). Procesos Creativos de Mercadotecnia: Ciudad de México: Ediciones Del Lirio-UPAEP.

Angenot, M. (2010). El discurso social. Los límites históricos de lo pensable y lo decible. Buenos Aires: Siglo XXI editores.

Arnould, E. y Thomson, C. (2005). Consumer Culture Theory (CCT): twenty years of research. Journal of consumer Research.31(4), pp.868-881. https://doi.org/10.1086/426626

Baumol, W. (2002). The free Market Innovation Machine. Analyzing the Grouth Miracle of Capitalism. Princeton: University Press.

Brunet, I. (2013). La iniciativa Emprendedora y la Innovación. Ciudad de México: FESE.

Brunet, I. y Pizi, A. (2012). Perspectivas de innovación y cambio técnico en Ciencias Sociales. Enlace Revista Venezolana de Información, Tecnología y Conocimiento. 9 (2), 79-94.

Cisternas, L. y Kast, P. (2014). Innovación Social. Una herramienta para mejorar la calidad de vida de las personas. Santiago: UDD- Instituto de Innovación Social, Fundación Kast.

Córdoba-Cely, C., Villamarín, F. y Bonilla, H. (2014). Innovación social: aproximación a un marco teórico desde las disciplinas creativas del diseño y las ciencias sociales. Revista de la Facultad de Ciencias Económicas y Administrativas, Universidad de Nariño.15(2), 30-34

Duarte, B. y Cortés, D. (2021). Diseño e Innovación Social como herramienta para el cumplimiento de Objetivos de Desarrollo Sostenible. En Proceedings INNODOCT/20. International Conference on Innovation, Documentation and Education. Editorial Universitat Politècnica de València. 711-718. https://doi.org/10.4995/INN2020.2020.11912

Eco, U. (2005). Tratado de semiótica general. Ciudad de México: Ediciones De Bolsillo.

Edquist, Ch. Y McKelvey, M. (1997). Small country innovation system, Globalization, change and policy in Asia and Europe. Cheltenham: Edward Elgar Publishing.

Enactus (2017). Nuestro propósito. http://enactusmexico.com.mx/

ENDEAVOR (2017). What we do. http://endeavor.org/approach/

Hahn, J. y Andor, L. (2013). Guide to Social Innovation. Eurepean Comission. http:// s3platform.jrc.ec.europa.eu/documents/20182/84453/Guide_to_Social_Innovation. pdf/88aac14c-bb15-4232-88f1-24b844900a66

Manzini, E. (2015). Design, when everybody designs: An introduction to design for social innovation. Cambridge: The MIT Press. 
Martínez, A. (2017). Las competencias educativas ¿una destrucción creadora del capitalismo?. En Delich, F. y Pablo, J. (Eds.). Economía, política y sociedad: Smith, Ricardo Marx, Keynes, Schumpeter, Preisch. (pp. 213-228). Córdoba: Comunicarte.

Observatorio Laboral (2012). Información por ocupaciones. http://www.observatoriolaboral.gob.mx/swb/es/ola/informacion_por_ocupaciones

Ortiz Nicolás, J.C. (2016). Diseñando el cambio. La innovación social y sus retos, Economía Creativa, otoño-invierno 2016 (6), pp. 9-35. https://dialnet.unirioja.es/servlet/ articulo? codigo $=5735360$

Rodríguez, A. y Alvarado, H. (2008). Claves de la innovación social en América Latina y El Caribe. CEPAL-Naciones Unidas.

San Martín, J. (2015). Antropología filosófica II. Vida humana, persona y cultura. Santiago de Chile: UNED.

Schumpeter, J. (2003). Capitalism, Socialism \& Democracy. London: Routledge.

UNESCO (2016). Puebla, Ciudad Creativa del Diseño para la UNESCO. Boletín de Prensa. http://www.unesco.org/new/es/media-services/single-view/news/puebla_ciudad_creativa_del_diseno_para_la_unesco/

UNESCO (s/f). Industrias creativas y culturales. http://www.unesco.org/new/es/santiago/ culture/creative-industries/

UPAEP (2012). Naturaleza y Destino de la UPAEP. Puebla: UPAEP.

\begin{abstract}
Design as a process, product, and action, has transformative potential in contemporary societies. In recent times, communities have faced challenges for intellectual capacities -logical and creative-, technological development, reinterpretation processes, and new approaches that improve people's quality of life and favor their interactions in specific contexts and circumstances. The design receives the call to participate in innovation processes to reduce the distance between the generation of ideas and their realization, highlighting the need to establish a social innovation frame that constitutes a frame of reference for training professionals. This research proposes a dialogue between what is written and what is said regarding design and social innovation. A socio-semiotic analysis of the concept of social innovation is developed through a literature review -the written- to later compare the speeches issued by an academic community of a local university -the said- based on a quantitative exploratory study. The main results of this dialogue are: a definition of social innovation and the identification of semantic values capable of constituting a code to establish a culture of social innovation and its praxis. The analysis is developed in a private university in Puebla, Mexico, through a socio-semiotic approach based on the fundamentals related to social discourse proposed by Marc Angenot and the code theory by Umberto Eco.
\end{abstract}

Key words: design -social innovation -cultural instauration - signification -social discourse - dialogue 
Resumo: $\mathrm{O}$ design como processo, produto e ação tem um potencial transformador nas sociedades contemporâneas. Nos últimos tempos, as sociedades têm enfrentado desafios que exigem o desdobramento da capacidade intelectual -lógica e criativa-, o desenvolvimento tecnológico, processos de reinterpretação e novas abordagens que melhorem a qualidade de vida das pessoas e favoreçam suas interações em contextos e circunstâncias específicas. A guilda do design é chamada a participar em processos de inovação para diminuir a distância entre a geração de ideias e a sua concretização, evidenciando a necessidade de se estabelecer uma cultura de inovação social que constitua um quadro de referência para a formação profissional. Esta pesquisa propõe um diálogo entre o que está escrito e o que é dito sobre design e inovação social, considerando os princípios do discurso social propostos por Angenot. Num primeiro momento, desenvolve-se uma análise sócio-semiótica do conceito de inovação social por meio de uma revisão da literatura - a escrita - para posteriormente comparar os discursos emitidos por uma comunidade acadêmica universitária a esse respeito-os ditos - com base em um quantitativo. Estudo exploratório. Como resultado desse diálogo, é oferecida uma definição de inovação social e destacados os valores semânticos capazes de constituir uma base de codificação para o estabelecimento de uma cultura de inovação social e suas práxis. A análise é desenvolvida em uma universidade privada da cidade de Puebla, México, por meio de uma abordagem sócio-semiótica baseada nos fundamentos relacionados ao discurso social proposto por Marc Angenot, e na teoria do código de Umberto Eco.

Palavras chave: design - inovação social - instauração cultural - significação - discurso social - diálogo

[Las traducciones de los abstracts fueron supervisadas por el autor de cada artículo] 